\title{
On the nonlinear coupling between micro turbulence and mesoscale magnetic islands in a plasma
}

\author{
W.A. Hornsby, A.G. Peeters, E. Poli, M. Siccinio, \\ A.P. Snodin, F.J. Casson, Y. Camenen, G. Szepesi \\ Centre for Fusion, Space and Astrophysics, \\ Dept. of Physics, University of Warwick, Coventry, U.K. \\ Max-Planck-Institut für Plasmaphysik, \\ Boltzmannstrasse 2, D-85748, Garching bei München, Germany
}

September 28, 2010

\begin{abstract}
The interaction between small scale turbulence (of the order of the ion Larmor radius) and meso scale magnetic islands is investigated within the gyrokinetic framework. Turbulence, driven by background temperature and density gradients, over nonlinear mode coupling, pumps energy into long wave length modes, and can result in an electrostatic vortex mode that coincides with the magnetic island. The strength of the vortex is strongly enhanced by the modified plasma flow response connected with the change in topology, and the transport it generates can compete with the parallel motion along the perturbed magnetic field. Density and temperature gradients inside the island are below the threshold for turbulence generation, and the anomalous transport inside the island is determined by turbulence spreading. A finite radial temperature gradient inside the island is observed to persist despite the fast motion along the field, and is related to the trapped particles which do not move along the field around the island. Consequences for the stability of the neo-classical tearing mode are discussed.
\end{abstract}

Multiscale dynamics receives a large amount of interest in the current literature, with a wide range of applications. A magnetized plasma is a particularly interesting system for studying the dynamical interaction since it supports waves and instabilities over a wide range of length and time scales. Furthermore, multiscale dynamics is crucial for the understanding of various phenomena in astrophysics as well as laboratory plasmas. In laboratory plasmas, the influence of mesoscale magnetic island structures on small scale turbulence and vice versa is expected to affect heat and particle transport properties $[1,2,3]$, although the exact mechanisms are poorly understood. Mesoscale, here, refers to 
a length significantly larger than the ion Larmor radius, but smaller than all other length scales (the gradient length of the temperature and density profiles, for instance). Mesoscale magnetic islands are formed through reconnection of the magnetic field, and are commonly obtained in both laboratory and astrophysical plasmas.

In this letter the interaction between small scale turbulence (order of the ion Larmor radius) and mesoscale magnetic islands is investigated through massive parallel computing and, to our knowledge, we present the first kinetic simulations that retain the electron dynamics. The letter concentrates on the tearing mode obtained in a tokamak plasma [4], with the results being directly applicable to existing experiments and, consequently, allowing for a validation through direct comparison. In the simplest picture the destruction of the nested magnetic surface topology through the formation of the island allows the plasma to flow along the field lines radially outward, reducing the energy confinement. However, the self-consistent electrostatic response connected with the island may attain large gradients resulting in a considerable sheared plasma flow. Such flows are known to stabilize small scale turbulence [5, 6] and, therefore, a confinement improvement is also possible. Indeed there is evidence that steep density and temperature gradients can form in the vicinity of island structures [7].

In this letter the state of the art gyro-kinetic model will be used to simultaneously describe both small scale turbulence and mesoscale magnetic structures. Simulations are performed with the GKW code $[8,9]$ which solves the MaxwellVlasov system in a flux tube geometry which follows the background magnetic field. The code is able to treat all species kinetically which is vital to the description of the island dynamics.

Fluxtube geometry is used to exploit the fact that turbulence is elongated along field lines, and have a short scale length perpendicular to the field[12]. The tube is of small (few gyro radii) radial and poloidal extent, while is extended along a field line of fixed pitch, i.e the number of toroidal turns per single poloidal turn. GKW uses field aligned Hamada coordinates [13, 9] that allow for an efficient numerical solution. The local approximation is used and therefore background quantities are assumed to be functions of radius only, which vary linearly.

The letter concentrates on nonlinear pumping of large scale vortex structures, as well as profile relaxation in the presence of the island. The timescale of these processes has been found to be short compared with the growth/decay of the island structure which is treated as static. The island structure is introduced in the model through an imposed perturbed vector potential parallel to the background magnetic field

$$
A_{\|}=\tilde{A}_{\|} \exp [\mathrm{i}(m \theta-n \phi)]
$$

where $\theta(\phi)$ is the poloidal (toroidal) angle, $\mathrm{n}(\mathrm{m})$ is the toroidal (poloidal) mode number, and $\tilde{A}_{\|}$is the amplitude of the perturbation. In the tokamak, any magnetic surface with a rational field line winding number, safety factor, $q=m / n$ is topologically unstable against the perturbation given above, i.e. an island structure in the magnetic field is formed around the rational surface.

Here, a large aspect ratio circular magnetic equilibrium will be assumed (the s- $\alpha$ model) for which the transformation to Hamada coordinates becomes $[9](s, \zeta)=(\theta / 2 \pi,[q \theta-\phi] / 2 \pi)$, where $s$ is the coordinate along the unperturbed 
magnetic field and $\zeta$ is the generalized toroidal angle. Assuming the winding of the magnetic field is resonant $(q=m / n)$ in the center of the computational domain $(\psi=r / R=\epsilon$, where $r$ is the radius of the magnetic surface, and $R$ is the distance of the center of the surface to the axis of symmetry), expanding $q$ up to first order in $\psi, q=m / n+\psi(\partial q / \partial \Delta \psi)$ gives $A_{\|}=\tilde{A}_{\|} \exp [2 \pi \mathrm{i} n(\zeta-s \partial q / \partial \psi \Delta \psi)]$. From this expression the wave vector of the island follows $k_{\zeta}^{I} \rho_{i}=2 \pi n \rho_{*}$, which is always the largest wavelength mode in the domain, where the ion Larmor radius $\left(\rho_{i}=m v_{t h} / e B\right.$ with $\left.v_{t h}=\sqrt{2 T_{i} / m_{i}}\right)$ is used for normalization and $\rho_{*}=\rho_{i} / R$ is the ratio of Larmor radius. As the code is pseudospectral in the perpendicular direction, the expression for the vector potential can be expanded in the Fourier modes used for the numerical implementation

$$
\begin{aligned}
\hat{A}\left(s, k_{\zeta}, k_{\psi}\right) & =\tilde{A}_{\|} N \exp \left(-\imath k_{\zeta}^{I} \zeta\right) \sum_{p=-\infty}^{\infty} \\
\exp \left(-\frac{(s N+p)^{2}}{L^{2}}\right) & \times \frac{\sin (\pi(s N+p))}{\pi(s N+p)} \exp \left(\imath p k_{\psi} \psi\right),
\end{aligned}
$$

where $\mathrm{N}$ is an integer that determines the radial mode spacing $\Delta k_{\psi}=(1 / N) k_{\zeta}^{I} d q / d \psi$. This expression contains an additional ad hoc damping at higher radial mode numbers (the term $\left.\exp \left[-(s N+p)^{2} / L^{2}\right]\right)$ in order to satisfy the periodic radial boundary conditions of the sheared flux tube domain. The results are unaffected provided the scale length $L$ is chosen to be large enough. In the simulations presented here this length is set to $L=2.0$ which is found to be of a sufficient length without affecting the physics. The contour lines of the perturbed vector potential given above summed with the background $A_{\| 0}=R B_{0} \psi^{2} / 2 q_{0}(1+\hat{s})$ form the surfaces traced out by the magnetic field and exhibit an island structure in a perpendicular plane to the field. The (half) width of the island is $w=2 \sqrt{q R \tilde{A}_{\|} / \hat{s} B}$. The perturbed vector potential is artificially imposed in the simulations of small scale turbulence driven by the free energy of density and temperature gradients. Since $\hat{A}_{\|}$is time independent the only effect it generates is the prallel motion along the perturbed field $v_{\|} \delta \mathbf{B} / B=v_{\|} \mathbf{b} \times \nabla A_{\|} / B$. Because the vector potential is imposed, Ampères law is not necessarily satisfied, and only the Poisson equation (quasi-neutrality) is treated self-consistently. The turbulence is essentially electrostatic with no other magnetic perturbations other than that of the island. Space limitations do not allow us to give all the model equations, but an exact description of all model equations, together with details on the implementation are given in Ref. [9].

Fig. 2 shows (a snapshot of) time traces of the heat flux averaged over the entire computational domain and broken up in different contributions. The parameters of these simulations are similar to those of the cyclone base case: normalized ion temperature gradient $R / L_{T}=6.9$, normalized density gradient $R / L_{N}=2.2$, inverse aspect ratio $\epsilon=0.19$, electron to ion temperature ratio $T_{e} / T_{i}=1$, safety factor $q=1.5$ and magnetic shear $\hat{s}=0.16$. The results in this paper are based on three sets of simulations, which will be referred to as $\mathrm{LL}, \mathrm{LH}$ and $\mathrm{HH}$, with the naming referring to the varying resolution/box size in the poloidal and radial direction. Unless explicitly stated otherwise, kinetic electron effects are kept at the true mass ratio of a Deuterium plasma. The simulations use $(\mathrm{LL}, \mathrm{LH}, \mathrm{HH})=(21,21,41)$ (positive) toroidal modes with $\Delta k_{\zeta} \rho_{i}=$ $(0.05,0.05,0.025)$, and $(101,167,167)$ radial modes (positive and negative) with 


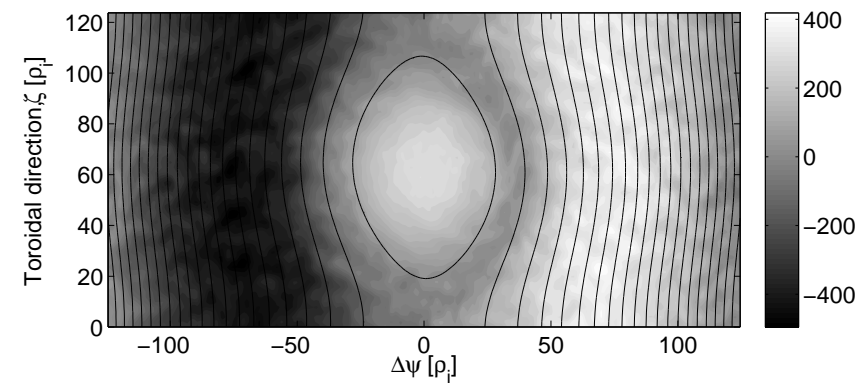

Figure 1: Normalized electrostatic potential $\left(\phi_{N}=e \phi / T \rho_{*}\right.$, case LH) in the plane perpendicular to the magnetic field (outboard midplane). Black lines represent the perturbed flux surfaces calculated from the total parallel vector potential.

$\Delta k_{\psi} \rho_{i}=(0.05,0.025,0.025)$. The wave vector of the island is always the longest wavelength on the grid, i.e. $k_{\zeta}^{I} \rho_{i}=(0.05,0.05,0.025)$. For a $m=3, n=2$ island which is resonant at $q=1.5$, this choice effectively determines $\rho_{*}=$ $\left(4 \cdot 10^{-3}, 4 \cdot 10^{-3}, 2 \cdot 10^{-3}\right)$, values that correspond to a large tokamak like ASDEX Upgrade. The radial width of the domain is chosen to be sufficiently large so that there is freedom for the flows around the island, and their perturbation by the island, to form. Temperature profiles return to their equilibrium values far before the boundaries which proves sufficient resolution is used.

The typical timescale for a particle of species $\mathrm{s}$ to traverse the field around the island $\left(\tau_{s \|}\right)$ and the timescale for perpendicular transport across the island $\tau_{s \perp}$ can be estimated to be

$$
\frac{\tau_{s \|} v_{t h i}}{R}=\sqrt{\frac{T_{i} m_{s}}{T_{s} m_{i}}} \frac{4 \pi q}{\left(k_{\theta}^{I} \rho\right) \hat{s}(w / \rho)} \quad \frac{\tau_{s \perp} v_{t h i}}{R}=\frac{1}{2 D_{s}^{N}}\left(\frac{w}{\rho_{i}}\right)^{2}
$$

where $D_{s}^{N}$ is the relevant transport coefficient in gyro-Bohm units $\left(D_{s}=D_{s}^{N} \rho_{i}^{2} v_{t h i} / R\right)$. For the parameters used in this paper $\tau_{i \|} \approx 196$ for $\mathrm{HH}$ and $\approx 97$ for $\mathrm{LH}$, $\tau_{e \|} \approx 3.3, \tau_{i \perp} \approx 40, \tau_{e \perp}=80$. Comparison of the timescales would indicate a moderate flattening for the ions for $\mathrm{LH}$, a slight flattening for $\mathrm{HH}$ and a complete flattening of the electron temperature in both cases.

The critical width above which sound wave propagation $[10,11]$ around the island will have an effect on the density is $w_{c} / \rho_{*}=R L n * q / \hat{s} \sim 20$ where RLn is the normalised ion density gradient. As such sound waves are likely to induce further flattening in the density gradients.

It has been suggested that sheared plasma flows connected with the island can stabilize turbulence and lead to improved confinement. The right panel of Fig. 2 shows that the time integrated total heat flux increases with the island size. (Although, as indicated by the horizontal lines, the error bars calculated as the standard deviation, do not allow for a confirmation of this increase at small island widths.) Since the perturbed distribution function is radially periodic, the temperature difference across the box is determined by the background temperature gradient and is independent of the island width. The increase in the heat flux, therefore, directly implies a confinement degradation with increasing island width. Time integrated (during statistical steady state) temperature profiles (background plus perturbed) along a radial chord through the O-point and X-point of the island are shown in Fig. 3. As expected, the presence of an 

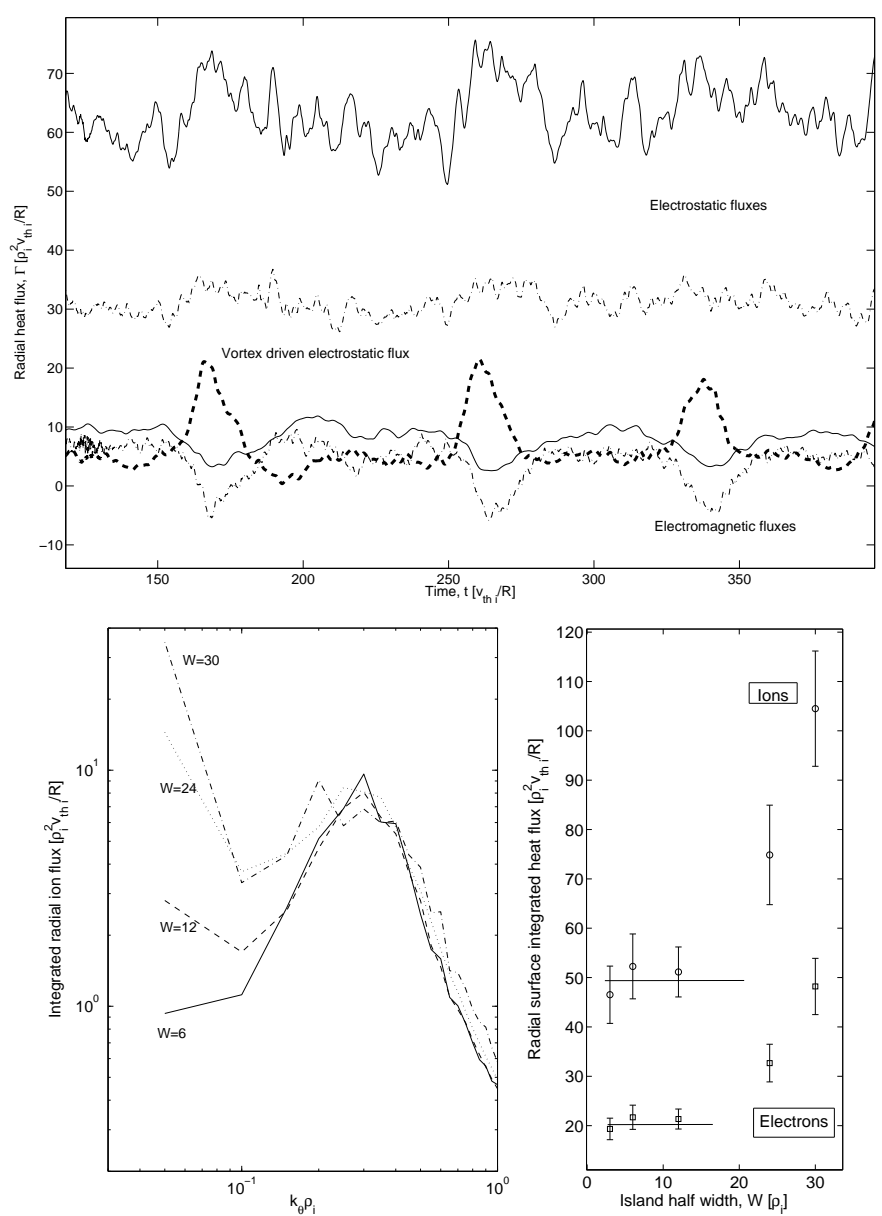

Figure 2: Left: time traces of the total electrostatic and magnetic flutter turbulent heat fluxes (for case LH) for ions (solid lines) and electrons (dashed lines) during the converged stage of a simulation with a magnetic island width of $w=24 \rho^{*}$. The thick dotted line gives the electrostatic heat flux generated by the modes with the same poloidal wave length as the island. Middle: The contribution to the electrostatic heat flux from the different toroidal modes as a function of the normalized poloidal wave vector for different island sizes (case LL). Right: Total heat flux in the ion and electron channel as a function of the island width (case LL). 
island structure leads to a flattening of the temperature profile inside the island. Fig. 3 also shows the temperature gradient as well as the turbulent fluctuations of the density $\delta n / n_{0}$. It can be seen that the gradient just outside the island structure, where the shear in the plasma flow is large, is strongly enhanced without a corresponding increase in the turbulence fluctuations. The sheared flows do stabilize the turbulence as previously reported [19], but the net effect of the island is nevertheless a degradation of confinement.

A striking observation shown in Fig. 2 is the magnitude of the electrostatic heat flux of the mode with a poloidal wave vector equal to that of the island. This contribution is observed to increase with the island size, and is enhanced by one to two orders of magnitude compared with the case without island. The reason for the increased transport at this wavelength is the formation of a large scale electrostatic vortex structure that coincides with the island as shown in Fig. 1. The vortex structure develops in the LL and LH cases for which $\tau_{i \|}$ is sufficiently small such that the temperature gradient is below the stability threshold of the ITG/TEM. For the HH case, with its larger $\tau_{i \|}$, the temperature gradient inside the island remains above the threshold and a turbulent flux is generated, although much reduced compared with outside the island. The $\mathrm{HH}$ case shows a much reduced vortex strength compared with the LL/LH case. It follows that this newly observed vortex structure is not due to a linear instability, but generated through the nonlinear pumping by small scale turbulence in a manner similar to the generation of the zonal flow. A finite level of fluctuations is present inside the island in the LL/LH cases through turbulence spreading [20, $21,22]$, i.e. the transport into the island of turbulent fluctuations driven outside the island. A large part of this turbulent spreading occurs in an unconventional way: fluctuations convect into the island in the drift direction via the X-point.

We note that the nonlinear pumping has previously been discussed in Ref. [14] which studies the linear stability of large scale modes in the presence of small scale turbulence. The vortex structure observed in our simulations, however, is different from the modes studied in Ref. [14] whose growth rates depend on the resistivity, which is zero in our collisionless simulations. We also note here that the variable nature of the vortex means that it is not the vortex predicted in [15] which comes from equilibrium considerations. The large scale potential structures outside the island, but NOT the vortex structure inside the island, are also found in simulations without turbulence and represent the equilibrium flow connected with the island [2]. The strength of the convective cell is largely enhanced by the modified flow response around the island analogous to the generation of zonal flows. In the case without an island, the magnitude of the potential for the $n=0$ 'zonal' mode is largely enhanced because the electrons can not balance the ion charge through a motion along the field. Similarly, in the case with an island the parallel electron motion can not balance an ion density that is constant along the perturbed magnetic surfaces. The result is a strong enhancement of the potential perturbation which is constant on the perturbed surface and, consequently, a large ExB flow along the perturbed surfaces. Indeed, simulations with an adiabatic electron density response $n_{e}=e n_{0} \phi / T_{e}$, which lack the physics of the stronger potential response, do not show a large vortex structure. The vortex is not constant in time, as shown in the left panel of Fig. 2, and the direction of rotation around the vortex can flip sign. One can note a clear anti-correlation between the radial heat flux generated by the vortex and the 'magnetic flutter', i.e. the heat flux due to the motion along 

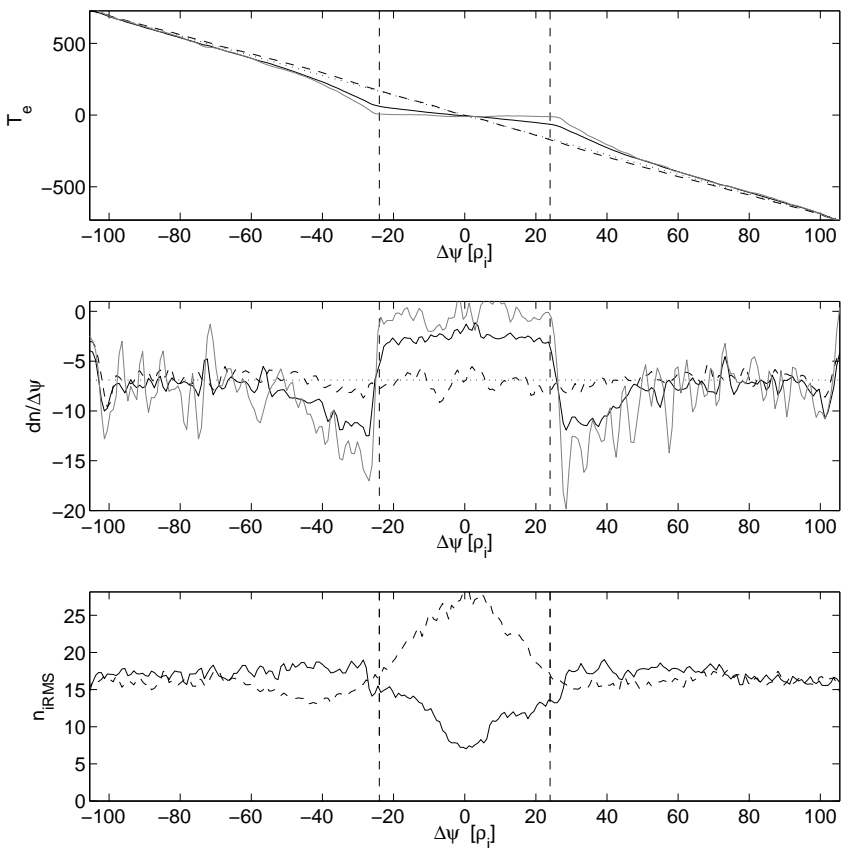

Figure 3: (for the HH case) Top panel shows the radial electron temperature profiles, black: Low field side, gray: High field side. Middle panel shows the corresponding electron temperature gradients. Bottom panel shows the RMS density fluctuation profile showing a decay of the fluctuation strength within the island. Dotted lines represent lines through the X-point.

the perturbed magnetic field that forms the island. At its maximum strength the transport due to the vortex largely exceeds the flutter flux, and on average its contributions to the heat flux is comparable. It is generally accepted that the fast transport along the magnetic field flattens the radial pressure gradient in the island, provided the perpendicular transport across the island is negligible against the parallel motion around the island [16]. The reduced pressure gradient inside the island plays an important role in magnetic confinement fusion since it leads to a reduction in the bootstrap current and can give rise to the so-called Neoclassical Tearing Mode (NTM) [17, 18]. The surprising result of our simulations is that profile flattening inside the island is not necessarily dominated by the fast flow along the field lines. The vortex structure leads to an enhancement of the profile relaxation inside the island, with smaller radial gradients being observed at stronger vortex strength.

Our simulations show that a modelling of perpendicular transport with a constant perpendicular heat conduction coefficient is largely inadequate. The heat conduction coefficients have been found to roughly follow the dependence of $\delta n / n$ and, consequently, transport in the island is reduced compared with the outer region. Inside the island the turbulent transport is affected by both the gradient as well as turbulence spreading / convection. In the sub-critical phase a vortex structure develops that reduces the perpendicular transport through shearing and is strong enough to compete with the parallel transport around the island. The transport coefficients are reduced in the O-point region while, they are strongly enhanced at the X-point.

The observed electron temperature gradients, in Fig. 3 are at odds with 
the expectations dervived from the time scales, showing a large radial electron temperature gradient inside the island. The presented simulations uncover an overlooked element in the theoretical description of the NTM. While the passing electrons move along the field lines resulting in a radial profile flattening, the trapped electrons do not, since the field line length around the island is much longer than $2 \pi q R$. The turbulent motion perpendicular to the field will, therefore, maintain a finite radial gradient in the trapped population. The temperature diagnostic used to generate Fig. 3 combines the background gradient with the integrated energy in the perturbed distribution function. Inside the island it combines the flat radial temperature of the passing particles with the finite gradient of the trapped particles. Since $\sim 50 \%$ of the particles are trapped, the observed reduction in the electron temperature gradient of roughly a factor two is consistent with a trapped particle distribution with a temperature gradient equal to the original equilibrium gradient. A demonstration that the finite temperature gradient in the simulations is due to the trapped particles is shown in Fig. 3, which additionally plots the temperature profile along a cord through the O-point on the high field side of the tokamak. No trapped particles reach the high field side position and, consequently the radial gradient is zero. Since it is the gradient in the trapped population that is responsible for the bootstrap current (see [23] and the references cited therein) we come to the astonishing conclusion that the bootstrap current is not reduced inside the island and the NTM would be stable. Of course, the argument above applies only in the limit of small (but finite) collisionality. De-trapping of electrons and ions, which occurs on a typical time scale $R \nu_{s} / r$, where $\nu_{s}$ is the collision frequency of species $\mathrm{s}$, will reduce the temperature and density perturbation in the trapped region, provided it occurs on a timescale shorter than the perpendicular transport through the island. For the NTM drive to become effective, the timescales of the two processes must be comparable, which results in a critical island width

$$
w_{c}=\sqrt{2 D r / R \nu_{e}} .
$$

We stress here that our simulations are collisionless and do not allow for the determination of the bootstrap current. The solution of a model problem including the effect of collisions, however, confirms the physical picture given above and will be published elsewhere [25]. Also this newly found effect does not contradict results in the literature [24] in which the kinetic equation has been solved for a finite collisionality without considering anomalous diffusion. In the latter case $w_{c}=0$ and the arguments above predicts a vanishing bootstrap current. Finally, for the determination of the bootstrap two additional mechanisms must be considered. First, anomalous transport inside the island is smaller than the transport outside the island and, hence, is less effective in maintaining the radial gradient. We note here that since it is the radial gradient of the trapped particle population that drives the trapped electron mode, there might be plasma parameters for which this drive is sustained inside the island, though this is not the case in the presented simulations. Second, the electrostatic vortex structure discussed above rotates the trapped particle population around the island. Indeed, we have found that when the vortex structure is sufficiently strong the temperature gradient of the trapped population is reduced. 


\section{Acknowledgments}

This work was jointly funded by the EPSRC via the S\&I grant and UKAEA/Euratom via Mobility. This work used resources on the HECToR supercomputer that were provided by the Engineering and Physical Sciences Research Council [grant number EP/H002081/1].

\section{References}

[1] S.-I. Itoh, Plasma Phys. Contr. Fusion 46, 123 (2004)

[2] H.R. Wilson, Plasma Phys. Contr. Fusion 51, 115007 (2009)

[3] F.L. Waelbroeck, Nucl. Fusion 49104025 (2009)

[4] J. Wesson, Tokamaks, Clarendon press - Oxford (Oxford, UK 2004)

[5] H. Biglari et al., Phys. Fluids B 21 (1990)

[6] R.E. Waltz et al., Phys. Plasmas 1, 2229 (1994)

[7] E. Joffrin et al., Nuclear Fusion 43, 1167 (2003)

[8] A.G. Peeters et al., Phys. Plasmas 11, 3748 (2004)

[9] A.G. Peeters et al., Comp. Phys. Comm. 180, 2649 (2009)

[10] B.D. Scott et al., Phys. Fluids 281 (1985)

[11] R. Fitzpatrick et al., Phys. Plasmas 13122507 (2006)

[12] M.A. Beer et al., Phys. Plasmas 2, 7 (1995)

[13] S. Hamada, Kakuyugo Kenkyu 1, 542 (1958)

[14] C.J. McDevitt et al., Phys. Plamsas 13, 032302 (2006)

[15] F.L. Waelbroeck et al. Phys. Rev. Lett. 87, 215003 (2001)

[16] R. Fitzpatrick, Phys. Plasmas 2, 825 (1995)

[17] J.D. Callen et al., Plasma Phys. Control. Nucl. Fusion Res. 2157 (1986)

[18] R. Carrera et al., Phys. Fluids 29899 (1986)

[19] E. Poli et al., Nucl. Fusion 49, 075010 (2009)

[20] X. Garbet, Phys. Plasmas 8, 2792 (2001)

[21] Z. Lin, Phys. Plasmas 11, 1099 (2004)

[22] V. Naulin, Phys. Plasmas 12, 122306 (2005)

[23] A.G. Peeters, Plasma Phys. Control. Fusion 42 B231 (2000)

[24] H.R. Wilson et al., Phys. Plasmas 3248 (1996)

[25] W.A. Hornsby et al., Phys. Plasmas, to be submitted 